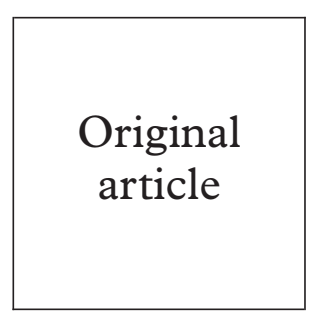

Department of

Epidemiology and

Public Health,

University of Leicester,

Leicester LE1 6TP, UK

$M$ Dixon-Woods

Department of

General Practice and

Primary Health Care,

University of Leicester,

Leicester General

Hospital, Leicester

LE5 4PW, UK

$\mathrm{T}$ Stokes

Department of Epidemiology and Public Health

B Young

Nuffield Community

Care Studies Unit,

Department of

Epidemiology and

Public Health

K Phelps

Department of

General Practice and

Primary Health Care,

University of Leicester,

Leicester General

Hospital

$\mathrm{K}$ Windridge

Leicestershire Health R Shukla

Correspondence to: Dr M Dixon-Woods md11@1e.ac.uk

Accepted for publication 10 May 2001

\title{
Choosing and using services for sexual health: a qualitative study of women's views
}

\author{
Mary Dixon-Woods, Tim Stokes, Bridget Young, Kay Phelps, Kate Windridge, \\ Rashmi Shukla
}

Objective: To explore women's accounts of choosing and using specialist services for sexual health.

Methods: A qualitative study involving semistructured interviews with 37 women screened for Chlamydia trachomatis attending genitourinary medicine and family planning clinics in an East Midlands health authority.

Results: In making the decision to seek help, women act on a range of specific prompts, including lay ideas about the significance of symptoms; their own behaviour; their partner's symptoms or behaviour; contact tracing; and health promotion. Some women do not seek help specifically in relation to sexual health but are identified as being at risk of sexually transmitted infections (STIs) during routine consultations. Important influences on women's choice of services include the lay referral system, "insider" knowledge of health services, referral by health professionals, and need to have alternatives to general practice care. Women's willingness to access services is mediated by psychosocial factors such as embarrassment. They may use smear tests as a legitimate opportunity to raise questions about sexual health. Women's priorities for services are that their feelings of stigma and embarrassment are managed appropriately; that staff communicate well and are sensitive to their emotions and comfort during tests and procedures; that they are "in control" when obtaining test results; and that confidentiality is preserved.

Conclusions: A detailed understanding of both lay people's response to symptoms or behaviour that indicates risk of an STI and their needs when using services should inform interventions aimed at encouraging secondary prevention of STIs.

(Sex Transm Inf 2001;77:335-339)

Keywords: consulting behaviour; lay beliefs; sexually transmitted infections

\section{Introduction}

Sexually transmitted infections (STIs) were ranked among the most important threats to public health in a recent survey of key clinical and public health professionals carried out by the UK Public Health Laboratory Service. ${ }^{1}$ The need for good control of STIs has become ever more pressing with recent evidence that people infected with an STI run much higher risks of contracting HIV through direct biological mechanisms. ${ }^{2}$ Primary prevention of STIs, involving changes in sexual behaviour, appears to have enjoyed limited success, ${ }^{3}$ and the role of secondary prevention, involving the detection and treatment of STIs, is therefore crucial. Secondary prevention requires that individuals at risk of STIs present themselves to appropriate services, and that those services be acceptable to them to encourage repeat use and "lay referral" of others. Little is known, however, about what makes people decide to seek professional attention for a suspected STI, or about their priorities when using services for sexual health. It is particularly important that these questions be addressed given that only a minority of those reporting risk markers for STI transmission attend clinics. ${ }^{4}$

Our paper reports an attempt to understand help seeking behaviour and views of specialist sexual health services in a sample of women attending family planning (FP) or genitourinary medicine (GUM) clinics. These services offer free open access diagnosis and treatment of STIs, and are central to efforts at STI control in the United Kingdom.

\section{Participants and methods}

There has been little previous research on help seeking in relation to STIs, and this area is likely to benefit from use of qualitative methods. $^{5-7}$ We obtained ethics committee approval to conduct a study involving semistructured interviews with women attending two GUM clinics (GU1 and GU2) and one FP clinic in an East Midlands health authority (population approximately 920 000).

\section{SAMPLING}

To avoid too diverse a sample, the study focused on women who had been screened for Chlamydia trachomatis, a condition for which all women attending the GUM clinics in the study and women who are considered to be at risk of an STI attending the FP clinic in the study were routinely tested. This approach allowed us to identify women with risk factors for STIs in both settings. Moreover, chlamydia was seen as an important condition around which to explore beliefs and behaviour in relation to sexual health services use, as it is the commonest curable STI in England and Wales, ${ }^{8}$ has important health consequences, and benefits from early detection and treatment ${ }^{9}$ but is often asymptomatic. However, interviews were not confined to discussions of this condition and the results presented below refer to a very wide range of STIs. 


\section{Key messages}

- Interventions to encourage women to use services for sexual health should emphasise behavioural cues and symptoms, not specific infections

- Smear tests provide an ideal opportunity for raising concerns about infections

- Specialist services for sexual health have a valuable role in meeting women's needs, particularly where general practice is seen as inaccessible or unacceptable

- Women want to be "in control" of receiving their test results

It was originally planned that a quota sampling approach would be used to reflect the range of demographic characteristics of women attending the clinics. In practice, all women who agreed to participate were interviewed, as the difficulties of recruiting women in such sensitive settings inhibited more purposive sampling. In the event, we were able to represent diversity of age and social class, but not ethnicity.

\section{DATA COLLECTION}

Recruitment to the study had to be handled carefully to protect women's confidentiality. Interviews were carried out on-site after women's clinic appointment without obtaining names or addresses. Women attended a GUM clinic (GU1 or GU2) or a family planning clinic (FP), or both GUM and FP, and this is indicated in the results section together with women's code names beginning with "W." The interviewers (KP and KW) approached women on a variety of times and days after appointments. Sociodemographic data and details of diagnosis (if available) were collected via a brief pre-interview questionnaire. Interviews were conducted using a checklist of topics to ensure that the same types of issues were covered with each participant. Topics included why women had sought help, what influenced their choice of services, and their views of the services they received. All interviews were tape recorded and fully transcribed.

ANALYSIS

The data were analysed using the constant comparative method. ${ }^{10}$ Analysis began with detailed indexing of the transcripts until recurring patterns were discerned which became the organising themes of the analysis. Systematic data analysis was assisted by NUDIST software using a themed coding frame developed initially by MDW and modified through discussion and independent analysis by TS, BY, and KP. The themes that emerged from the data are presented here together with illustrative quotations.

\section{Results}

There were 37 participants in the study. This figure was sufficient to achieve theoretical saturation $^{11}$ : no new themes emerged from the analysis after the 22nd interview and later interviews served to augment and confirm themes identified earlier in the analysis. Participants were aged between 15 and 53 and were from socially diverse backgrounds. They included 12 students, four clerical/secretarial workers, three homemakers, three sales assistants, two teachers, and other occupations including one novelist, a commercial sex worker who employed other sex workers, and several women whose occupations were associated with caring or health professions. Three of the women were black and the remaining 34 white; it proved impossible to recruit women of south Asian ethnicity to the study.

WHAT PROMPTS WOMEN TO USE SERVICES FOR SEXUAL HEALTH?

Women identified five possible prompts for help seeking specifically in relation to sexual health, as listed in table 1 .

The symptoms that prompted women to seek help included pain and discomfort (especially on intercourse), frequent urination, bleeding, rash, abdominal pain, discharge, and "cystitis" or "thrush" that did not seem to clear up. In interpreting the significance of these cues, women experienced considerable uncertainty. They tended to invoke a lay symptomatology that often did not recognise the potential importance of the symptoms, saw them as initially attributable to "innocent" explanations such as "natural" changes, and assumed that symptoms would spontaneously resolve. Very few women formulated a hypothesis as to the specific infection or problem they might have. It was only as symptoms became increasingly intolerable, inexplicable, or frightening that the decision to consult was made:

I've left it and left it and left it 'cos I thought that basically, you know, I was just sore and then having sex again I hadn't had time to recover and I was still sore ... um but it was getting to the point where you know it's causing me problems ... going to just have to tell my boyfriend no all the time. (W35, aged 20, GU2)

For some women, the trigger for the consultation came from their partner's symptoms, diagnosis of their partner's symptoms, or diagnosis of their partner's ex-partner. Women were also prompted to consult by discovery of their partner's behaviour, including "sleeping around," or having sex with sex workers.

I had a lying, cheating bastard of a boyfriend... I found him in bed [with another woman] and an automatic reaction was, right, I'm going to get myself checked. (W25, aged 26, GU2)

Consultations could be prompted by women's recognition that their own sexual behaviour had been risky, particularly where it involved unprotected sex with "risky" partners, including drug users. Some women reported being traced as contacts. Others used GUM clinics as a means of health maintenance, or preventive health activity following risky behaviour, often so that they could assure a new partner that they were free of infection.

Table 1 Prompts for help seeking

Own symptoms

Partner's symptoms

Partner's behaviour

Own behaviour

Health maintenance, including being traced as a contact 
No symptoms at all. I had no idea ... it was just for my own peace of mind really. You know the end of a relationship. Fust to make myself feel sort of like if I met somebody else I can say . . .. (W15, aged 24, GU2)

Finally, over a quarter of those in the sample (10 women) had not been seeking help for actual or suspected STIs in the first instance, but were in fact seeking help for other health matters, including smear tests or contraception, when they were tested for STIs. Smear tests were used by women as a legitimate opportunity to raise questions about symptoms that were bothersome but too embarrassing to raise in other contexts or did not seem to warrant a separate consultation:

I happened to be in for a smear. I wouldn't have made an appointment to go in just about a little bit of soreness. For a long time I thought it was just dryness... (For) about 3 weeks we were very careful and very slow and made sure that I was ready before we started and it still hurt and that's when I thought that I would mention it when I go for my smear test. (W37, aged 18, GU1)

WHAT INFLUENCES WOMEN'S CHOICE OF SERVICES FOR SEXUAL HEALTH?

Women found out about specialist services for sexual health in three ways: the lay referral system; "insider" knowledge of health services on the part of the health workers or those who knew health workers; and contact tracing or referral by a health professional. For most, the lay referral system was perhaps the most important source of information. Once women had used the service themselves, they often became self appointed ambassadors, encouraging others to use the service also:

I send everyone here because I come here myself. It's quite nice. (W5, aged 25, FP)

Twenty three of the women in the sample had used specialist services for sexual health more than once. Many women preferred to bypass general practice entirely. Previous users of FP tended to go there when they first suspected a sexual health problem, and were often then referred on to GUM clinics in cases of infection. Many women also went directly to a GUM clinic, especially if they had used it before, or made an effort to find out about it to avoid using general practice. Some said that they would not have sought help at all if alternatives to their general practitioner (GP) had not been available; they perceived specialist services as more relaxing environments or as offering higher standards of clinical care:

I just went to (my GP) and I said "oh I think it's thrush" and he just went "oh well, it is then" and gave me Canesten and it wasn't and it was no help. (W13, aged 17, FP)

Some women feared judgmental attitudes on the part of GPs, and four reported that they or their friends had had negative experiences, including being shouted at or accused of promiscuity by GPs on previous occasions when they sought help for sexual health issues. Women who perceived that they had a friendly relationship with their GP were also uncomfortable about consulting him/her, and the perceived anonymity of specialist services was a key factor in their choice of service:

He knows your family and you kind of think when you are sitting there, he knows my mum, he knows my brothers and stuff like that and knowing that you're at things like that. (W9, aged $15, \mathrm{FP})$

The importance of confidentiality and anonymity were recurring themes in the women's accounts. Many did not want the issue of an STI to appear in their medical records, and they felt that confidentiality was more likely to be observed in specialist services than at their own GP surgery.

Speed and ease of access were valued by many women, who referred to difficulties in getting an appointment with GPs even if they were happy to consult them about a sexual health problem. The need for appointments systems and access arrangements at specialist services to be clear was emphasised. In addition to issues of physical accessibility, psychosocial issues such stigma and fear of pain and "the unknown" were powerful mediating influences on women's use of services, particularly for GUM compared with FP clinics. For example, one woman reported that one of her employees, a commercial sex worker, refused to visit a GUM clinic with a stuck vaginal sponge after sexual intercourse with seven clients:

"I'm not going down there, it's degrading, they stick your legs in them bloody stirrups." (W31, aged 28, GU1)

WOMEN'S EXPERIENCES OF SEXUAL HEALTH SERVICES

Once women had accessed a sexual health service, they were generally satisfied overall. However, they distinguished particular aspects of the service as areas of particular satisfaction or dissatisfaction, including those listed in table 2 .

The quality of the "atmosphere" was a key priority for women:

...coming here I was nervous at the beginning and sort of terrified . . .um . . .but they have made me very welcome, sort of well not welcome I suppose but they've had a very friendly atmosphere around them. Um ... which did make me relaxed. (W34, aged 18, GU1)

The physical environment, including the waiting area, was not a cause for particular comment, probably because these were of a reasonably high standard in the services in the study. By contrast, the social aspects of waiting were very important. Women described how they both monitored and were monitored by the others in the waiting room, potentially normalising their visit for some women, while alienating others:

Table 2 Dimensions of satisfaction

Atmosphere and environment

Staff-patient relationships and communication

Management of tests and treatment

Management of stigma and embarrassment Confidentiality 
...you haven't got to be worried about it, 'cos you're not on your own and, well, nobody had two heads, nobody looked, like, strange at all. I mean obviously nobody judges you. (W24, aged 18, GU1)

...the patients were all so young and here's me thinking oh my god I feel like you know. I felt like well I was old enough to be some of them's mother you know and I thought . . .oh sugar! (W23, aged 41, GU2)

A key priority for women attending specialist services was the management of their feelings of stigma and embarrassment. It was vital to them that clinic staff did not appear judgmental, and that staff dealt with the same type of problem routinely.

They understand, it's not like you don't come in and you feel really dirty 'cos you've got something. Like they treat it every day. (W29, aged 18, GU1)

Women's experiences of tests and treatment for STIs formed an important element of their experiences and judgments of the service. Most women reported feeling nervous, exposed, and vulnerable.

...it's, oh God, I haven't shaved my legs, do my feet smell? (W19, aged 21, GU2)

Women varied in the discomfort and pain they experienced while the tests were carried out, and communication appeared to have an important role in mediating these experiences.

"This usually hurts a bit, it's over quickly" is nice, I can cope with that, but "this may be a little bit itchy" is not very useful when you're feel as if your head's going through the ceiling. (W30, aged 53, GU1)

Not as bad as I thought it was going to be but I think it was a lot down to how nice the doctor was, just really put me at ease... (W13, aged 17, $\mathrm{FP}$ )

Many women had not realised that they would be tested for a range of STIs, thinking that only one type of infection would be screened for, and were surprised or taken aback, talking of the need to "psych themselves up" for tests. Women who had been opportunistically screened-when accessing contraceptive services, for example-were also taken aback by testing. Most women emphasised the need to be kept well informed, with clear explanations from staff, particularly in relation to explaining medical procedures, treatments, prognosis, and issues around contact tracing. Sensitivity to information needs was a priority; women wanted to feel that they could ask questions of staff and were keen to have written information available. Women disliked receiving what they saw as conflicting information; four mentioned in particular getting different information from different professionals. The role of the health adviser in GUM clinics was seen as particularly important in good communication.
The method of obtaining results of tests was very important to women, as was speedy diagnosis. There were several possible methods of getting the results: phoning the clinic, visiting the clinic or GP, getting a letter from the clinic, or being phoned by the clinic. Women much preferred having control and being allowed to phone in themselves to get results. Eleven women specifically expressed strong dislike of getting letters, and two reported that they did not like being phoned by clinics. Being contacted in these ways was seen as risking confidentiality, and as unsupportive and potentially anxiety creating.

(I) had a phone call from this woman who told me I had chlamydia. I sat in my front room with my window open. Told me on the telephone I am from the Family Planning, I am just ringing to tell you you have got chlamydia. I thought it was a bit bizarre to tell me on the telephone to be quite honest, thought it was a bit weird. (W6, aged 21, FP/GU1)

\section{Discussion}

Our study offers accounts from 37 women from diverse social backgrounds and age groups of their behaviour and views in relation to services for sexual health. It was disappointing that no women of south Asian origin were recruited to the study, despite south Asians forming $10 \%$ of the population in the health authority area where the study was conducted. The reasons for the lack of participation include apparently lower use of specialist services, especially GUM, by women from this ethnic group, and their reluctance to participate in this type of research when approached. ${ }^{12-14}$

Our study offers significant insights into how women interpret and respond to sexual health symptoms and behaviour, and what influences their decision to consult a health professional (table 1). It suggests that women do not seek help from specialist services for sexual health on the basis of suspicions about specific infections. Instead, they respond to a range of bothersome, inexplicable or frightening symptoms, or to behavioural cues. Women in our study were diagnosed with a number of different STIs, but had not formed hypotheses in advance of seeking help as to the exact infection that they might have, possibly because of poor knowledge about STIs. ${ }^{15}$

These insights could inform the design of interventions to increase the use of sexual health services. Many current interventions, such as posters and leaflets, tend to focus on specific infections. We would suggest that future interventions should instead focus on the significance of particular types of symptoms and types of behaviours, including partners' symptoms and behaviours. Women should also be encouraged to use specialist services as a means of health maintenance, and to raise concerns about STIs when attending for a cervical smear in whatever setting.

Women's decisions to access specialist services for sexual health were influenced by three factors: the lay referral system; "insider" knowledge of health services; and contact tracing or referral by a health professional. It is 
clear that specialist services play an extremely valuable part in meeting the needs of women with actual or suspected STIs. Our study confirms earlier suggestions ${ }^{16}$ that users of specialist services often reject GP services for sexual health for reasons associated with their relationship with their GP, their views of the quality of clinical care, and their need for confidentiality and accessibility, although some women may well prefer GP services for other reasons. Previous research has also suggested that users of sexual health services tend generally to be satisfied $^{5}$ and our study has confirmed this as well as identifying more precisely women's priorities for services. Again, the benefits of specialist services, as perceived by women themselves, should be emphasised in interventions to encourage access to services; it is important that their priorities, rather than the priorities of providers, are highlighted.

If high quality sexual health services are to be delivered then the views of users must be taken into consideration. ${ }^{17}$ We have identified which dimensions of satisfaction users view as being of particular importance in services for sexual health (table 2). Key priorities were that services should address women's feelings of stigma and embarrassment and need to be "in control" of their test results. Given the role of women themselves as "ambassadors" of the services, services need to ensure that they address these priorities if they are to encourage repeat use and lay referral. These findings can also be used to inform the design of future questionnaire based studies and audits.

Sexually transmitted infections represent a public health problem that needs to be brought under control. The UK government is currently consulting on an integrated sexual health and HIV strategy, and it is important that this includes consideration of how to promote secondary prevention through improved uptake of specialist services for sexual health. We would suggest that interventions to encourage greater use of specialist services, and the planning and organisation of services, needs to be guided by detailed understanding of lay people's response to the behavioural and physical symptoms that indicate risk of an STI, and their needs when using services.

Funding: Medisearch.

Competing interests: None declared.

We should like to thank all the women who agreed to be interviewed and Drs Paul Schober and Gillian Wandless for their help in allowing the study to be conducted. We should also like to express our appreciation to Jane Whitehouse for her help in to express our appreciati
typing the transcripts.

Contributors: TS had the original idea for the study and TS,
Cong the transcripts.

Contributors: TS had the original idea for the study and TS,
MDW, RS, and BY designed the protocol; TS and MDW conMDW, RS, and BY designed the protocol; TS and MDW con-
ducted the literature review; KP and KW recruited the patients and conducted the interviews; MDW carried out the analysis and was assisted by TS, BY, and KP; MDW and TS wrote the paper and all authors contributed to the final draft of the paper.

1 http://www.phls.co.uk/whoweare/ovcdrep.htm

2 Fleming DT, Wasserheit JN. From epidemiological synergy to public health policy and practice: the contribution of other sexually transmitted diseases to sexual transmission of HIV infection. Sex Transm Infect 1999;75:3-17.

3 Adler M. Sexual health-a Health of the Nation failure. BMF 1997;314:1743.

4 Johnson AM, Wadsworth J, Wellings $\mathrm{K}$, et al. Who goes to sexually transmitted disease clinics? Results from a national sexually transmitted disease clinics? Results from a nation
population survey. Genitourin Med 1996;72:197-202.

population survey. Genitourin Med 1996;72:197-202.
5 Evans D, Farquhar C. An interview based approach to seeking user views in genitourinary medicine. Genitourin Med 1996;72:223-6.

6 Warwick I, Aggleton P, Homans H. Constructing commonsense-young people's beliefs about AIDS. Sociology of Health and Illness 1988;10:213-33.

7 Calnan M. The patient's perspective. Int 7 Technol Assess Health Care 1998;14:24-34.

8 Simms I, Catchpole M, Brugha R, et al. Epidemiology of genital Chlamydia trachomatis in England and Wales. Genitourin Med 1997;73:122-6.

9 US Department of Health and Human Services (CDC). Recommendations for the prevention and management of Chlamydia trachomatis infections. Morbidity and Mortality Weekly Report 1993;42:1-38.

10 Green J. Commentary: grounded theory and the constant Green J. Commentary: grounded theory and
comparative method BMF 1998;316:1064-5.

11 Muphy E, Dingwall R, Greatbatch D, et al. Qualitative research methods in health technology assessment: a review of the literature. Health Technol Assess 1998;2(16).

2 Balarajan R. The ethnic populations of England and Wales: the 1991 census. Health Trends 1992;24:113-16.

13 Fenton K, Johnson AM, Nicoll A. Race, ethnicity and sexual health BMF 1997;314:1703-4.

14 Bowler I. Problems with interviewing: experiences with service providers and clients. In: Miller G, Dingwall R, eds. Context and method in qualitative research. London: Sage, 1997.

15 Devonshire P, Hillman R, Capewell S, et al. Knowledge of Chlamydia trachomatis genital infection and its consequences in people attending a genitourinary medicine quences in people attending a gent. Sex Transm Inf 1999;75:409-11.

16 Lenaars PEM, Rombouts R, Kok G. Service attributes and the choice for STD health services in persons seeking a medical examination for an STD Soc Sci Med 1994;38: 363-71.

17 Hopkins A, Gabbay J, Neuberger J. Role of users of health care in achieving a quality service. Qual Health Care 1994; 3:203-9. 